\title{
Réflexions sur la valeur de la liberté : Mary Ann Shadd, la conscience anti-esclavagiste et la fonction de la lettre ouverte
}

A Meditation on the Value of Liberty: Mary Ann Shadd, Anti-Slavery Conscience, and the Labor of the Public Letter

\section{Suzanne B. Spring}

Traducteur : Sivan Cohen-Wiesenfeld

\section{(2) OpenEdition} Journals

Édition électronique

URL : http://journals.openedition.org/aad/1004

DOI : $10.4000 /$ aad. 1004

ISSN : 1565-8961

Éditeur

Université de Tel-Aviv

Référence électronique

Suzanne B. Spring, "Réflexions sur la valeur de la liberté : Mary Ann Shadd, la conscience antiesclavagiste et la fonction de la lettre ouverte ", Argumentation et Analyse du Discours [En ligne], 5 | 2010, mis en ligne le 20 octobre 2010, consulté le 23 septembre 2019. URL : http:// journals.openedition.org/aad/1004; DOI : 10.4000/aad.1004

Ce document a été généré automatiquement le 23 septembre 2019.

\section{(†)

Argumentation \& analyse du discours est mis à disposition selon les termes de la licence Creative Commons Attribution - Pas d'Utilisation Commerciale - Pas de Modification 4.0 International. 


\title{
Réflexions sur la valeur de la liberté : Mary Ann Shadd, la conscience anti-esclavagiste et la fonction de la lettre ouverte
}

\author{
A Meditation on the Value of Liberty: Mary Ann Shadd, Anti-Slavery Conscience, \\ and the Labor of the Public Letter
}

Suzanne B. Spring

Traduction : Sivan Cohen-Wiesenfeld

Mary Shadd chérissait la liberté. Sans doute n'est-ce guère surprenant de la part d'une femme noire libre qui atteignit l'âge adulte durant les deux dernières décennies de la réforme anti-esclavagiste, dans les années 1840 et 1850 . Sa rhétorique pratique était fondée non seulement sur la Liberté comme principe "allant de soi », mais aussi sur la Constitution qui garantissait à chaque individu le droit à la liberté de conscience sous toutes ses formes : religieuse, politique, matérielle, discursive. Affirmer son droit à la parole était cependant un exercice de la liberté contesté dans la mesure où, dans ces décennies précédant la Guerre civile, l'« esclavocratie » au pouvoir continuait à en priver des centaines de milliers de gens de couleur libres - et à dénier catégoriquement ce droit constitutionnel à des millions d'autres maintenus en esclavage ${ }^{1}$.

Dans son sens élargi, la liberté en tant que valeur s'est constituée, en ces temps qui ont précédé la guerre civile, au sein de ce qu'on pourrait considérer comme « un système de croyances que l'on prétend valoir aux yeux de tous » et dans lequel les valeurs républicaines étaient " traitées comme des faits ou des vérités " (Perelman 1958 : 101). La nature idéographique de la Liberté signifiait qu'elle pouvait être interprétée de manières largement différentes. Dans le Sud et la plus grande partie du Nord, par exemple, elle était conçue comme la latitude des Etats de se gouverner eux-mêmes et de protéger la propriété qui incluait la possession d'esclaves et les intérêts des citoyens, mais aussi d'étendre le système politique et économique de l'esclavage vers les 
territoires de l'Ouest (McGee 1988). Pour la plupart des abolitionnistes, en revanche, la liberté n'était pas seulement un concept de gouvernement abstrait ou un ensemble de droits; c'était un objectif, en l'occurrence, l'abolition de l'institution politique de l'esclavage. Cependant, Mary Ann Shadd ne partageait pas la conception de la plupart de ses alliés sur le rôle de la liberté dans la réforme anti-esclavagiste. Pour elle, la liberté n'était pas seulement une fin, elle était aussi un moyen. En d'autres termes, la liberté ne signifiait pas seulement la fin de l'esclavage, elle s'incarnait de manière individuelle et collective dans l'homme libre et ses relations économiques, politiques, religieuses et sociales. Pour Shadd, cette Liberté comme objectif conceptuel et pragmatique pouvait trouver sa meilleure réalisation dans la culture et l'incarnation, à travers la connaissance institutionnelle et l'échange discursif, de ce qui était déjà doué de liberté : la capacité humaine universelle de perception, d'analyse et de critique.

La conception de Shadd de la liberté comme moyen et comme fin aurait probablement été admise par d'autres réformateurs abolitionnistes si elle avait établi une hiérarchie claire entre les deux, en privilégiant la fin immédiate d'un système violent et injuste au détriment $\mathrm{du}$ développement de rapports justes entre hommes libres. On trouve souvent ce genre d'hiérarchie dans les systèmes de logique des valeurs traditionnels, qui supposent une distinction « des fins et des moyens, les fins ultimes correspondant à des valeurs absolues»(Perelman 1958: 368). Cependant, les efforts réformistes de Shadd furent marqués par son refus de réifier les hiérarchies de valeurs et peuvent se caractériser par cette remarque due aux philosophes de la rhétorique Chaïm Perelman et Lucie Olbrechts-Tyteca :

dans la pratique, il existe une interaction entre les buts que l'on poursuit et les moyens mis en œuvre pour les réaliser. Les buts se constituent, se précisent et se transforment, au fur et à mesure de l'évolution de la situation dont font partie les moyens disponibles et acceptés ; certains moyens peuvent être identifiés à des fins, et peuvent même devenir des fins, en laissant dans l'ombre, dans l'indéterminé, ce à quoi ils pourraient servir $(1958: 368)$.

On peut soutenir que pour Shadd, en tant que femme qui se sentait engagée par la promesse de sa nation, la «fin» laissée dans "l'ombre, dans l'indéterminé», était l'impératif constitutionnel pour le «Peuple» de «former une Union plus parfaite». L'Unité devait se structurer au travers de grammaires intégrées incluant la "Justice ", la «Paix intérieure » et les "Bienfaits de la Liberté », qui fonctionnaient également comme moyens de faire évoluer le présent déjà " parfait » de la nation vers son «plus parfait » avenir ou sa "Postérité» (Constitution américaine de 1787). A travers un tel réseau de valeurs primordiales, la nation pourrait anticiper ses propres possibilités à travers les relations réciproques, voire en apparence tautologiques, entre la fin et les moyens.

Compte tenu de l'engagement particulier de Mary Ann Shadd à l'égard de la Liberté, la compréhension et la mise en œuvre des relations co-constitutives entre fins et moyens devenait un impératif moral. Il s'agissait, selon elle, d'une besogne publique qui exigeait des hommes libres - «qu'ils aident à façonner l'opinion publique sur la question de l'esclavage en insistant sur une ligne de conduite d'obédience biblique dans les relations humaines; de la part des gouvernements envers les individus, et de la part des individus pour préserver leur liberté ». M. Shadd pensait que seule une telle relation tripartite permettrait « de comprendre clairement les énormités d'un système tel que l'esclavagisme américain et ses analogues, le despotisme russe et l'oppression italienne et autrichienne, et de les détruire » (Provincial, 25 mars 1854, souligné dans l'original). 
Façonner l'opinion publique, ajoutait Shadd, signifie s'engager dans une critique publique à travers l'échange discursif: "prendre la précaution d'exposer aux [personnes] possédant ou non un sens moral et religieux les caractéristiques immorales, irréligieuses [et] révoltantes de tout système d'oppression même modéré, est une mesure indispensable à la préservation de la liberté ». En d'autres termes, elle affirmait que pour préserver la liberté, les citoyens doivent exposer au grand jour tout système d'oppression, car ils sont tous reliés entre eux, quelque bénins qu'ils puissent paraître et où qu'ils se trouvent dans le monde. Martin Luther King reprendra un argument semblable un siècle plus tard: "Toute injustice, où qu'elle se produise, est toujours une menace pour la justice dans le monde entier. Nous sommes pris dans un inextricable réseau de relations mutuelles, sous la chape d'une même destinée. Tout ce qui affecte l'un directement affecte tous les autres indirectement » $(1963: 85)$.

Le parallèle entre Shadd et King est significatif au-delà du sentiment partagé qu'il reflète. King affirme ce principe relevant des droits civils sur le même mode rhétorique d'échanges mutuels que Shadd avait adopté comme forme principale de son discours public : la lettre ouverte. Il existe, aux Etats-Unis, de nombreux précédents d'emploi de la lettre ouverte comme genre réformiste et comme forme de rhétorique politique. Comme Stephen Howard Browne l'a montré, la lettre ouverte a joué un rôle pivot dans l'établissement d'une politique collective radicale pendant la période prérévolutionnaire de la nation. C'est ce qu'illustrent bien les Lettres d'un fermier de Pennsylvanie aux habitants des colonies britanniques de John Dickinson en 1767-1768, qui "projeta une image des colons, bien que dispersés géographiquement, comme un peuple lié par des convictions mutuelles, des valeurs partagées et un désir collectif du bien public », ayant pour but « de créer les conditions sous lesquelles la révolution était imaginable» (2009: 518). Les lettres publiques ${ }^{2}$ circulèrent même plus largement à la période postrévolutionnaire, le Congrès cherchant à unifier la nouvelle nation à travers l'institution politique d'une poste nationale. Destinée à l'origine à la diffusion de la connaissance publique à travers la circulation des rapports gouvernementaux, des journaux et des pamphlets et étant donné l'accord des journaux d'autoriser la reproduction illimitée des articles et des lettres ouvertes, la portée de la poste, et donc également des débats locaux sur l'esclavage, a pu s'étendre, comme le découvrit Tocqueville, des échelons les plus élevés des administrations jusqu'au plus profond des forêts de la frontière américaine $(1835: 172-180 ; 369)^{3}$. A une période de pré-guerre civile pendant laquelle la collectivité nationale était davantage tournée vers la discorde que vers la concorde, Mary Ann Shadd se joignit aux milliers d'épistoliers qui utilisèrent ce forum public effervescent et transformèrent dramatiquement les EtatsUnis en une manifestation particulièrement politisée de la « République des Lettres ».

On verra que le souci de la liberté de la conscience individuelle et collective - vivre en tant qu'hommes libres devenant la valeur absolue et le but ultime - était une motivation majeure des lettres ouvertes et du discours de Mary Ann Shadd, aux EtatsUnis et au-delà. Employant la lettre ouverte comme une forme de rhétorique antiesclavagiste, Shad travaillait à susciter chez son public une liberté de conscience susceptible d'articuler (terme pris ici dans son sens étymologique d'articulare - « diviser en parties distinctes » et « relier ») ses nombreux principes, valeurs et allégeances ${ }^{4}$. En d'autres termes, pour Shadd, la valeur de la liberté et son exercice dans la lettre ouverte consistait dans la possibilité de s'engager dans l'expression de sa conscience à travers des échanges souvent conflictuels avec les autres, et anticipés comme tels échanges façonnés par l'attente et l'exigence d'une réciprocité dans l'expression des 
libertés individuelles et collectives. Il s'agit d'une approche de l'interaction sociale et de la rhétorique politique dans laquelle les hommes tirent pleinement avantage de leur capacité humaine de critique collective, et où les individus peuvent et doivent s'engager pour transformer leurs institutions à travers ce que $\mathrm{C}$. Wright Mills nomme une « transvaluation des valeurs » $(1959: 8)$ entretenue par le discours et, bien sûr, les lettres d'examen de conscience.

\section{« Insensible aux frontières » : 'articuler' le Peuple}

Au début de l'année 1849, à l'âge de vingt-cinq ans, Mary Ann Shadd écrivit sa première lettre ouverte à Frederick Douglas, rédacteur du North Star. Elle venait de quitter West Chester, en Pennsylvanie et de retourner à Wilmington, dans le Delaware, pour fonder une école pour enfants noirs libres. La promotion raciale à travers l'éducation, tout comme l'action politique à travers la presse, devait lui sembler quasi inévitable ; elle faisait partie de l'héritage de la famille activiste dans laquelle elle avait grandi ${ }^{5}$. Shadd débuta cette première incursion dans la vie publique en éliminant toute distance susceptible de l'éloigner des préoccupations des communautés noires libres: «Bien qu'originaire d'un Etat différent, je suis, pour tout ce qui concerne notre peuple, insensible aux frontières » (23 mars 1849). Les frontières qu'elle franchissait, dans cette lettre ouverte et les nombreuses autres qui suivirent, étaient à la fois géographiques et idéologiques, et sa subtile référence à "notre peuple » était une tactique pour se positionner, elle, une femme libre de l'Etat esclavagiste du Delaware, au même niveau de leadership que Douglass, ancien esclave vivant à présent dans l'Etat nominalement libre de New York.

D'une certaine façon, la lettre de Shadd est représentative des nombreuses « lettres à la rédaction » du milieu du dix-neuvième siècle qui prévalaient dans les colonnes des journaux de l'époque. La lettre à Douglass était un trope du discours public. Cependant, il $\mathrm{y}$ avait un niveau d'adresse supplémentaire dans la mesure où il s'agissait avant tout d'une réponse à une précédente lettre publiée dans le North Star par Henry Highland Garnet, un autre réformiste anti-esclavagiste respecté. La lettre de Garnet appelait à une "Convention chrétienne" susceptible de réunir les trente mille personnes de couleur dispersées dans les comtés du nord de l'Etat de New-York. Douglass avait approuvé cet appel, admettant l'urgence que constituait «la dégradation morale et religieuse des gens de couleur de l'ouest de l'Etat de New-York »; mais il l'avait modifié pour inclure "tous les hommes et femmes, saints et pêcheurs", et non les seuls Chrétiens (19 janvier 1849). Shadd chercha à élargir cette modification de l'appel, non seulement en étendant sa portée géographique au-delà des frontières de l'Etat, mais également en refusant ses normes de politesse. Plutôt que le consensus, elle proposait le dissensus : «Nous n'avons réalisé que peu d'efforts pratiques. En tant que femme de ce peuple, je ne vois pas la nécessité de nos réunions particulières, si nous ne faisons rien. Nous devons agir davantage ", insista-t-elle, « et parler moins » (23 mars 1849). Sa lettre suggérait d'abandonner les signes extérieurs de la classe moyenne ainsi que le matérialisme pour "devenir producteurs» au lieu de rester de simples consommateurs. Elle exigeait qu'on passe à des préoccupations politiques indépendantes du «clergé corrompu, qui sape tous nos moyens et, en compensation, nous inculque l'ignorance qui serait un devoir, et la superstition qui serait la vraie religion »; enfin, elle les enjoignait à profiter de toutes les occasions pour parvenir « au 
savoir de l'homme blanc, dont nous pouvons glaner une grande partie dans les relations qui se créent au sein des réunions publiques etc.». Ce savoir public et politique, affirmait Mary Ann Shadd avec autorité, était «ce dont nous avons le plus besoin intellectuellement, et ce dont nous ressentons le plus le manque » (23 mars 1849).

10 Ce que Shadd cherchait à 'inculquer' à ses lecteurs par une critique directe, voire incessante, c'était de travailler en commun à l'engagement civique, d'acquérir «le savoir de l'homme blanc » qu'on pouvait obtenir dans les réunions publiques. C'était un travail que les communautés noires libres devaient entreprendre pour prendre leur place au sein du peuple américain, en particulier sous l'égide de la valeur républicaine de l'autonomie. Shadd pensait que le "clergé corrompu " interdisait au peuple de s'engager dans les affaires publiques, et elle somma l'Eglise de "ne pas empêcher [le peuple] de se prendre en charge » : le succès final viendrait "lorsque nous utiliserons les bons moyens", et le moyen à utiliser était de "faire advenir nous-mêmes le changement que nous souhaitons, sans attendre que les blancs du pays le fassent » (23 mars 1849). En d'autres termes, la fin poursuivie, l'obtention de la Liberté, nécessitait que les gens de couleur libres emploient les moyens appropriés, en l'occurrence l'usage de leur liberté. En fait, à travers l'utilisation de la lettre ouverte, Shadd appelait ses lecteurs à développer une conscience critique des relations qu'ils entretenaient non seulement entre eux, mais aussi avec des systèmes politiques et économiques complexes, tout comme avec le système de croyances personnelles sur lequel reposait leur communauté religieuse et sociale. Toute rupture potentielle de ces relations ou toute forme d'isolation sociale ou politique des Noirs libres, résultant de leur assujettissement à l'Eglise plus qu'au gouvernement de l'Etat, ou de leur confiance dans les Blancs plus que dans les Noirs libres, mettaient en danger la place de "notre peuple » au sein " du Peuple », et donc la cause anti-esclavagiste.

11 Les relations réciproques que Shadd cherchait à établir entre les réalités discursives, économiques, politiques et matérielles, positionnaient sa rhétorique, selon les termes de Nathan Stormer sur les capacités d'articulation de la rhétorique, "comme une imbrication exquise, voire infernale, de signification, de matière et d'action ", dans laquelle « la rhétorique est toujours "en devenir" à travers la production mutuelle des corps et des langages et leur configuration dans des ordres complexes" $(2004: 262)$. Cette approche conférait une valeur particulière à la dynamique des échanges. Dans la mesure où Mary Shadd accordait aux individus le pouvoir de mettre en œuvre les relations entre le peuple, les institutions et la réalité matérielle, la réciprocité devenait pour elle le principe structurant de toute interaction sociale. De plus, puisque les auteurs de lettres ouvertes pouvaient potentiellement mettre en œuvre des relations allant bien au-delà de leurs destinataires locaux d'origine, ils devenaient des orateurs politiques susceptibles, comme le souligne Lloyd Bitzer, de constituer un pouvoir " dans des situations qui présentent des problèmes, des crises, des obstacles, ou autres types de situations appelant une intervention, que [les orateurs politiques] tentent de modifier en adressant des messages à des auditoires médiateurs, c'est-à-dire à des auditoires qui possèdent un pouvoir suffisant pour modifier ces situations » (1981: 14, souligné par moi). L'anticipation et l'exigence de réciprocité dans des situations de conflit déstabilisent donc toutes les tentatives de centralisation d'un orateur désireux de contrôler unilatéralement le discours ou l'institution; le bon orateur politique serait en conséquence celui qui cherche à constituer des collectivités, non seulement pour perpétuer ses propres valeurs ou croyances, mais aussi pour mobiliser le pouvoir d'un 
auditoire réceptif d'effectuer un changement social et de s'engager à son tour comme orateur.

La critique sans compromis devait donc être plus productive que destructrice. Dans un pamphlet circulant à la même période que sa première lettre dans le North Star, Shadd présentait sa conception de la lettre ouverte :

Ressentant, que mon destin est celui de mon peuple, c'est un devoir envers moimême... d'exposer toutes les faiblesses, de dénoncer toute habitude qui contribue à prolonger notre oppression. Je dois donc tenter de susciter votre réaction, non par la raillerie ni le ridicule, mais comme quelqu'un qui, par consentement, sinon par participation effective, a apporté sa contribution à une prise de parole directe et sans peur (cité dans North, 8 juin 1849).

En se présentant comme complice de relations sociales conflictuelles, Shadd érige le débat - le débat transparent - en moyen d'éveiller la conscience sur l'état actuel des choses, dans le but de faire participer les individus à la création de ce qu'ils désirent : la liberté collective. Comme un correspondant de L'Etoile du Nord le faisait observer, la réception du pamphlet de Shadd fut cependant mitigée : il fut largement lu dans la région de Philadelphie, et pourtant peu de gens souhaitaient l'acheter. Le correspondant supposait que l'unique défaut de ce pamphlet était «de trop dire la vérité, en montrant en particulier la condition de notre peuple sous son vrai jour » (8 juin 1849). Shadd, cependant, ne se découragea pas. Si le désaccord et la différence résultaient inévitablement des efforts à nouer des relations avec les autres, seule une articulation irréprochable des problèmes et des obstacles - dans le cadre d'un échange verbal accessible au public - pouvait donner aux gens la liberté de travailler à la constitution d'une collectivité prête à délibérer de ces inévitables différences et conflits.

\section{« L'opposant honnête » et le travail de dissension}

L'année qui suivit l'entrée de Mary Shadd sur la scène du discours public, le Congrès fit passer le Compromis de 1850 en adoptant cinq projets de loi, dont l'un était la loi sur les esclaves en fuite. Celle-ci renforçait la loi de 1793 en imposant la restitution des esclaves en fuite à leurs propriétaires, et l'étendait en demandant aux citoyens ordinaires d'aider à la capture des fugitifs et en refusant l'accès des tribunaux aux hommes et femmes rendus à l'esclavage. La capacité du groupe parlementaire proesclavagiste du Congrès à diminuer les libertés des citoyens blancs comme des Noirs, non seulement frappa de stupeur les milieux abolitionnistes, mais plaça également les Noirs du Nord, anciens esclaves ou nés libres, sous une menace constante d'arrestation. Les abolitionnistes réagirent à cette loi par un regain vigoureux d'unité en faveur de l'abolition immédiate de l'esclavage, aidant les esclaves à gagner la liberté grâce au Chemin de fer clandestin, et construisant des communautés où les Noirs libres pourraient pleinement faire usage de leur liberté.

Le Canada était le havre où, pour beaucoup, ceci semblait le plus réalisable, où la citoyenneté était accordée sur une base égalitaire, au moins en termes de race, si bien que l'émigration vers le Canada devenait une alternative à prendre sérieusement en compte pour les Noirs du Nord ${ }^{6}$. Plus tôt, en 1851, Mary Ann Shadd y avait elle-même émigré et avait commencé à y travailler au développement des libertés à sa portée, fondant une école pour l'éducation des enfants canadiens "sans distinction de 
couleur ", associant sa participation active aux réunions publiques à une prodigieuse production et diffusion de lettres publiques, à la fois au Canada et aux Etats-Unis (American Missionary Association, 27 novembre 1851). Bien que Shadd ait publié des lettres dans des journaux abolitionnistes comme The Voice of the Fugitive, établi au Canada, The Pennsylvania Freeman, Frederick Douglass' Paper (FDP) et The Liberator, publiés aux Etats-Unis, ce n'est qu'après avoir fondé The Provincial Freeman qu'elle sentit qu'elle avait les moyens « d'avoir l'oreille du public » et que «sa personnalité, son poids et ses capacités " seraient mises à contribution «dans un journal se proclamant la voix des fugitifs » (cité dans Rhodes, 62 ; Frederick Douglass' Paper, 17 décembre 1852).

Dans son premier numéro du 4 mars 1853, Shadd et les cofondateurs du journal définirent The Provincial Freeman avec emphase comme étant «un JOURNAL franchement et ouvertement ABOLITIONNISTE ». En dénonçant « le droit de propriété sur d'autres êtres humains et [...] tout système ou sentiment lié à cette idée, ou allant dans ce sens ", et en prenant la défense des "réfugiés des plantations du Sud", The Provincial Freeman devait leur indiquer « les moyens et les mesures qui permettront une amélioration et une évolution susceptibles d'en faire des travailleurs indépendants, pourvoyant à leurs besoins, justifiant la liberté impartiale dont ils jouissent ici, et contribuant par leur énergie et leur labeur à la prospérité de leur pays d'adoption ». Shadd considérait la lettre ouverte comme un des principaux «moyens » mis à la disposition des réfugiés, et la première page du numéro inaugural mettait en valeur trois lettres : la première était une introduction du rédacteur, co-fondateur du journal, Samuel Ringgold Ward qui présentait les but du Provincial Freeman et déclarait que son œuvre anti-esclavagiste exercerait « un effet-ricochet sur l'esclavage de la république voisine [...], qui ne pourra être ni renié ni contrecarré» (4 mars 1853). Sa lettre était suivie de deux autres qui avaient déjà été publiées dans The Pennsylvania Freeman, l'une rédigée par Ward et la seconde par C. C. Foote, présentant chacun sa position, respectivement à l'encontre et en faveur de la Refugee Home Society (RHS), une association d'aide fondée en 1851 qui proposait des terres et un soutien matériel aux fugitifs arrivés au Canada et était financée en grande partie par des associations américaines philanthropes bénévoles. Entre autres accusations, Ward soutenait que la RHS encourageait la dépendance à travers l'institution d'un "système de mendicité " pour les réfugiés, les réduisant à " une sorte de serfs » (4 mars 1853). Il affirmait que la RHS était «non seulement pro-esclavagiste dans sa doctrine quant à la capacité des Noirs de détenir et gérer la propriété, mais se trompait dans ses déclarations concernant la condition des fugitifs ». Shadd était d'accord avec lui.

17 L'approche adoptée par Shadd pour fournir un cadre à la lecture de l'échange WardFoote, cadre qui fera de la lettre publique, selon les termes employés par Shirley Wilson Logan, « un lieu d'éducation rhétorique » $(2008,8)$, est d'un intérêt primordial pour le présent article. Introduisant les deux lettres, Shadd remarquait: «nous publions l'ensemble pour que nos lecteurs puissent juger la controverse dans toute son ampleur » (Provincial, 4 mars 1853). Elle fit suivre ces lettres d'une longue postface, affirmant qu'elle "juge bon d'ajouter quelques notes à la communication ci-dessus ", qui prit la forme d'un commentaire interprétatif en dix points, chacun d'eux correspondant à un numéro placé à l'intérieur des lettres originales. Le premier point affirmait : « Le regroupement d'épithètes [tel que pratiqué par M. Foote], dont chacun est détaché de son contexte où il est qualifié par d'autres termes, est un procédé rhétorique rarement employé par un opposant honnête. Dans cet exemple, la manoeuvre est injuste envers M. Ward, comme le lecteur s'en apercevra en lisant la 
lettre de celui-ci ». Elle continue son analyse serrée du texte, démontrant à son lecteur que Foote ne tient pas compte des arguments de son interlocuteur et n'avance, quant à lui, aucun argument valide. Après avoir signalé les fautes de logique de Foote, comme le fait de décontextualiser, de procéder à des généralisations abusives et d'esquiver les accusations de Ward, Shadd renvoie son lecteur aux lettres afin qu'il effectue sa propre analyse rhétorique et « juge par lui-même ». Ce travail d'analyse suggère l'incarnation de ce qu'elle appelle l'« opposant honnête » : celui qui, dans le débat public et dans ses discours, est capable de " juger la controverse " grâce à ses aptitudes à appréhender et évaluer la validité des arguments de l'autre et qui, dans le contexte de l'échange, répond en produisant ses propres arguments justes et raisonnables, basés sur une logique claire et des preuves satisfaisantes.

18 Mary Ann Shadd ne se contente pas de guider ses lecteurs par son approche rhétorique, elle fait également elle-même le travail d'un "opposant honnête ». Au cours des mois qui ont précédé la création du Provincial Freeman, The Liberator avait publié une série d'échanges, comprenant cinq lettres ouvertes, chacune prenant alternativement la défense ou critiquant la RHS. Le numéro du 24 décembre 1852 présentait en première page une lettre de C. C. Foote en faveur de la RHS, que Shadd critiqua dans une lettre datée du 12 janvier 1853 et publiée dans The Liberator le 4 mars 1853 : « La lettre du Rév. C. C. Foote ne réfute rien ", affirmait-elle, «[...] mais, avec une merveilleuse ingénuité, un singulier mélange de fausses affirmations, de comparaisons curieuses, de recommandations éculées en faveur du Fugitives' Home dues à des messieurs qui n'ont jamais examiné de près son système en fonction, d'hypocrisie et de Case de l'Oncle Tom, elle entend passer devant une communauté intelligente " (4 mars 1853). Pour que la "communauté intelligente» cesse d'être abusée, Mary Ann Shadd proposa une réfutation point par point de la lettre de Foote, et y annexa le protocole d'une réunion publique tenue à Windsor par les réfugiés " pour s'exprimer, énergiquement, contre le Refugees' Home Society, et la lettre de M. Foote ». Le protocole rapporte que Mary Shadd a été élue pour répondre à la lettre de Foote " au nom du peuple ", et que les « motions adoptées au cours de la réunion l'avaient été à l'unanimité » à l'encontre de Foote et décidant «de multiplier les réunions, et d'être entretemps vigilant pour empêcher le succès de la forme d'oppression qu'on voit apparaître dans la Refugees' Home Society ». Le protocole était signé de quarante-six noms d'émigrants, et Mary Shadd s'assura de bien distinguer quarante-trois réfugiés de trois Noirs libres, afin de réfuter l'accusation de la RHS selon laquelle les critiques à leur encontre étaient le fait des seuls Noirs libres comme Shadd, auxquels la RHS refusait son aide et qui agissaient par avidité et jalousie plus que par solidarité avec les émigrants réfugiés.

La lettre de Shadd dans le Liberator devait produire, au moins en partie, l'effet désiré. Le rédacteur en chef William Lloyd Garrison admettait que soupeser les deux côtés de la "Controverse du Canada» était devenu une entreprise "plus que complexe». Il affirmait qu'il ne pouvait pas douter des membres de la RHS, dont il avait longtemps cru «qu'ils n'avaient d'autres motifs que le bien-être des malheureux fugitifs »; pourtant, il concédait que "Miss Shadd, appuyée par un nombre considérable de réfugiés, semble présenter des arguments plausibles du côté opposé ». L'argumentation que Shadd avançait était rigoureuse dans les preuves avancées et dans son raisonnement, et comme elle adoptait la forme de la lettre ouverte, elle mettait en relief non seulement la présence de l'auteure, elle-même une émigrée, mais aussi des quarante-six autres émigrés qui s'étaient rassemblés en public pour dénoncer Foote et le système de la RHS. Cependant, même confronté aux «arguments plausibles» de 
Shadd, Garrison, lui-même renommé pour sa rhétorique "incendiaire ", finit par conclure : "Déplorant profondément ces dissensions, et peu enclins à prendre partie pour ou contre le projet des Réfugiés, tout ce qui nous reste à faire à cette distance est de permettre impartialement aux deux côtés de s'exprimer dans nos colonnes, en espérant que la controverse soit rapidement close » (4 mars 1853).

Shadd, cependant, n'allait pas clore le débat: le premier numéro du Provincial Freeman parut le même jour que celui du Liberator contenant l'échange Ward-Foote et le commentaire de Shadd, et celle-ci poursuivra sa critique de Foote et de la RHS à travers des lettres ouvertes, les siennes propres et celles des autres, dans The Provincial Freeman tout au long des années cinquante. Pourtant, la controverse arriva bientôt à sa fin dans le Liberator, même si cela ne fut que par le silence du journal. The Liberator ne reproduit pas l'analyse rhétorique persuasive de l'échange de lettres Ward-Foote effectuée par Shadd. En revanche, il publia l'annonce, déjà parue dans le Frederick Douglass' Paper, d'un meeting public des partisans de la Refugee Home Society à Sandwich, au Canada, ayant pour but « de dénoncer M. Ward et ses amis, et de condamner la proposition de fonder un autre journal [à savoir le Provincial Freeman de Mary Ann Shadd] consacré aux intérêts des gens de couleur " (23 avril 1853). Il ne réimprima pas le commentaire que le Frederick Douglass' Paper avait inséré dans l'annonce : «Pour notre part, nous saluons la création dans notre voisinage d'une douzaine de journaux "consacrés aux intérêts des gens de couleur", car il n'est pas trop de ces publications pour faire face aux besoins de toutes les classes » (13 avril 1853). Au lieu de cela, The Liberator présentait simplement la motion du meeting du RHS presque comme s'il s'agissait du dernier mot dans la controverse entre Shadd et Foote : «Croyant résolument que "l'union fait la force" et la seule manière de nous élever [...], nous réprouvons donc, et condamnons les actes [de Windsor] comme étant hautement injurieux pour notre cause » (23 avril 1853). Sans doute les partisans de la RHS avaient-ils probablement - et inconsciemment - été motivés autant par leurs propres intérêts économiques que par leur croyance sincère dans le narratif des "fugitifs mourants de faim ", mais ils étaient profondément pénétrés de la valeur de «l'Union ». Pour les partisans de la RHS comme pour Garrison, «l'Union» fonctionnait comme un front uni des abolitionnistes s'opposant aux institutions et au pouvoir esclavagistes. L'insistance de Shadd sur la dissension et sur la mise en évidence du paternalisme incontesté du mouvement abolitionniste, entretenu, selon elle, par un préjugé racial esclavagiste qui lui était inhérent, menaçait cette unité. Or, même la force de son argument ne put perturber l'engagement des partisans en faveur de cette valeur. Chaïm Perelman et Lucie Olbrechts-Tyteca expliquent pourquoi les communautés positionnent si souvent l'unité en haut de leur échelle de valeurs: «Etre d'un avis qui s'écarte de celui de tous les autres, c'est rompre une communion sociale fondée, croit-on - et le plus souvent à juste titre - sur des données d'ordre objectif [...]. [Cette unanimité] toutes les sociétés tiennent à l'assurer, car elles en connaissent la valeur et la force» (1962: 75-76). Pour protéger leurs efforts de persuasion morale pacifique, le mouvement abolitionniste devait s'unir contre les puissants propagandistes de l'esclavagisme, de même que contre les fréquentes menaces de sécession du Sud, qui défiaient l'unité même de la nation. Bien que Shadd ait soutenu ce même but de l'unité du camp anti-esclavagiste, elle refusait de placer l'union au-dessus de la liberté d'expression de la conscience individuelle, en partie parce que mettre une union restreinte au-dessus de l'interrelation des valeurs républicaines ne permettrait pas de réaliser son but ultime. 
21 En effet, dans un article intitulé «L'union » dans ce premier numéro de The Provincial Freeman, elle soutenait que la tendance vers la «désunion » caractérisait en fait la famille humaine (4 mai 1853). Elle affirmait que

certains craignent que les différences d'opinion existantes au sein de notre peuple, concernant certaines affaires publiques, s'avèrent désastreuses. Nous ne partageons pas ces craintes. [...] nous confessons librement que nous ne souhaitons pas l'union aux dépens de la pensée et de l'action libres et indépendantes. Au risque d'être accusés de séparatisme, nous ne demandons à personne de nous suivre aveuglément, ni d'être d'accord avec nous; et Celui qui nous a crées sait que nous ne suivrons aucun démagogue, ni blanc ni noir, ni sur la doctrine ni en pratique, dans une direction que notre jugement et notre conscience désapprouvent [...]. L'union est souhaitable - plus que souhaitable - et digne de tolérance, de pardon, d'abnégation et de charité ; mais l'union acquise aux dépens des principes moraux est trop chère payée. Il vaut mieux rester seul avec ses principes, que de suivre la foule en compromettant ses principes (4 mars 1853).

Ce que Perelman et Olbrechts-Tyteca nomment une « communion sociale » n'était pas, pour shadd, une apparence superficielle d'unité, ni une absence de discorde. Sa version de l'union était plutôt celle d'un «contrat social " rousseauiste entre les individus se fondant sur le principe des Lumières selon lequel tous sont également libres et également liés par la responsabilité de cultiver et faire progresser l'union libre et égalitaire à laquelle ils ont consenti. Si un individu décide, à travers ses facultés « de jugement et de conscience ", que ce principe moral est compromis, il est de son devoir de faire connaître cette erreur, même si sa critique s'écarte entièrement de ce que le groupe a déterminé comme étant juste. Pour Shadd, « rester seul fidèle à ses principes » ne signifiait pas qu'un dissident se place à l'extérieur de la communauté sociale. Cela signifiait que s'il percevait qu'un compromis s'établissait sur un but jugé important, il lui incombait d'utiliser le moyen de la critique pour rectifier l'orientation prise par le groupe, lui permettant de d'œuvrer à nouveau avec intégrité et de réaliser l'idéal de l'union.

23 Shadd était véritablement ingénieuse: sans doute n'y avait-il pas de forme plus appropriée à cette critique que la lettre ouverte, qui se distinguait par sa structure de médiation et son grand rayon géographique dû au réseau de distribution postale des journaux. Même la communauté des esclavagistes reconnaissait que ce réseau permettait au discours anti-esclavagiste de « circuler à travers les veines et les artères de la société » et d'offrir ainsi aux orateurs anti-esclavagistes un accès au corps politique et partant un pouvoir pour influencer l'opinion publique (Charleston Courier, 30 août 1835).

24 L'opinion publique que Shadd cherchait à influencer était constituée principalement par l'auditoire anti-esclavagiste. En termes d'échange épistolaire destiné au débat autour de la RHS, elle cherchait à montrer que la structure même de la RHS en tant qu'institution d'assistance, même si l'on tenait compte des relations plutôt distantes et apparemment bonnes entre les donateurs américains et les bénéficiaires canadiens, nécessitait de perpétuer le narratif des "pauvres réfugiés", selon l'expression de Garrison - des gens affamés, dépendants et sans défense -, impliquant des relations de discrimination hiérarchiques au sein de la communauté, plutôt que de mettre en place un narratif énonçant ce qu'ils étaient réellement: des hommes libres possédant le droit, en tant que sujets britanniques, d'embrasser et de mettre en pratique une pleine citoyenneté canadienne. Compromettre les relations libres et égales de cette collectivité anti-esclavagiste revenait, aux yeux de Shadd, à réaliser l'union «aux 
dépens des principes moraux ». Aussi exigeait-elle la ré-articulation de deux des valeurs les plus importantes de la collectivité, l'unité et la liberté, et l'application de ces valeurs étroitement liées de manière à ce que tous les membres de cette collectivité travaillant à ses fins spécifiques soient en possession des structures mentales aptes à les tenir en alerte contre toute concession à ces principes. Une partie du travail de l'« opposant honnête » anti-esclavagiste était donc de négocier le "contrôle social » qui est, comme l'a bien vu Michael McGee, « essentiellement un contrôle de la conscience, une influence a priori que des prédispositions acquises exercent sur les agents humains qui jouent le rôle de "pouvoir" et de "peuple" dans une transaction donnée " (1980: 88). Les conventions formelles de ce puissant genre politique intégraient les structures de réception et de production de l'échange, une configuration permettant de faire de la reconnaissance mutuelle une responsabilité : dans les relations qu'on entretient avec les autres, avec les institutions et avec le langage lui-même, mais permettant aussi d'ouvrir de nouvelles voies pour obtenir et préserver pour la collectivité la Liberté comme idéal, comme moyen et comme fin.

\section{La conscience anti-esclavagiste et la « transvaluation des valeurs »}

Le choix de Mary Ann Shadd pour la dissension fait partie d'une tradition plus large aux Etats-Unis. Comme le soutient la philosophe politique Martha Nussbaum, la dissension basée sur la liberté de conscience a son origine non pas dans la période révolutionnaire et dans la Constitution, mais dans l'Amérique coloniale du dix-septième siècle, à travers la figure de Roger Williams. Comme Shadd, Williams avait émigré pour trouver la liberté ; cependant, en tant que Puritain, la libération qu'il recherchait se référait aux persécutions religieuses menées par la couronne d'Angleterre ${ }^{7}$. Pour Nussbaum le plus grand apport de Williams consiste dans son «insistance sur la quête profondément subjective de la conscience individuelle, et la valeur sans prix de la liberté dans cette quête ", autant que dans sa conception et sa défense de la conscience comme faculté " caractérisée en partie par ce qu'elle fait - ses motifs, ses recherches et les expériences émotionnelles de désir qui y sont liées - et en partie par les sujets dont elle traite - des questions fondamentales, ou de sens fondamental». Nussbaum conclut: "C'est la faculté, non son but, qui est à la base du respect politique, et en tant qu'elle s'adresse à une "capacité" qui exige d'être à la fois développée et exercée » (2008:169). Comme on l'a vu, Shadd insiste également sur la " quête subjective » et sur « la valeur sans prix de la liberté dans cette quête", et elle recommande et enseigne l'usage actif de la conscience comme faculté analytique de raisonnement. Elle se démarqua aussi de ceux avec qui elle était en apparence alliée, lorsqu'elle comprit qu'ils allaient contre les injonctions « du jugement et de la conscience » (Provincial, 4 mars 1853).

Cependant, Shadd se différencie de Williams par l'acception large qu'elle confère à la liberté de conscience, étendant son sens des croyances religieuses subjectives aux croyances, actions et relations sociales et politiques d'une communauté. Plus précisément, le traité de Williams, La doctrine sanglante de la persécution, affirme la nécessité de ce qu'il nomme, dans le langage puritain, l'« auto-condamnation », ce qui suggère un parallèle avec Shadd en ce que cette auto-condamnation fait partie de la faculté de la conscience "à travers laquelle le peuple de Dieu dans tout son éveil reconnaît combien ils ont peu écouté l'examen de leur propre conscience» (1867: 89). 
William développe une acception de la vigilance communautaire au sein de laquelle la conscience de chaque individu est responsable de son propre "examen"; Shadd affirme le pouvoir de la conscience subjective tout en l'articulant à l'examen de la conscience collective, par le peuple. Lorsque la quête d'un peuple a pour but une telle articulation, la « valeur sans prix de la liberté » intègre à la fois un moyen, une fin et un principe directeur. Tel est l'essentiel du potentiel de la lettre ouverte comme genre relevant de la conscience : c'est une forme qui peut structurer les examens nécessaires au peuple pour qu'il devienne, pour ainsi dire, conscient de la conscience, et dans laquelle la valeur ultime devient l'échange lui-même.

27 Je voudrais illustrer par un dernier ensemble d'échanges la manière dont Shadd a cherché à faciliter ce travail. Lorsque le Provincial Freeman démarre au début du printemps 1854, Shadd prend un moment pour enseigner à ses correspondants potentiels l'importance de la critique : «Traitez aussi sévèrement que vous le souhaitez des mesures et des motivations des autres journaux ou rédacteurs, si vous considérez qu'ils ont tort. Si vous ne le faites pas », prévient-elle, « nous le ferons» (22 avril 1854). Ses lecteurs répondirent si généreusement que sa «table gémit littéralement sous le poids de leurs faveurs "; celles-ci comprenaient des lettres relatant des expériences et formulant des critiques sur les formes de la mendicité et les diverses structures d'oppression; des récits d'émigrants et de visiteurs présentant des narratifs complexes du peuple noir au Canada et aux Etats-Unis; des courriers sur des problèmes urgents adressés à des hommes libres ou des communautés anti-esclavagistes sur le continent américain et à l'étranger; et même des lettres d'éloge et de blâme concernant The Provincial Freeman ou Shadd elle-même.

En raison de la circulation du Provincial Freeman à la fois au Canada et aux Etats-Unis, l'un des problèmes dont les lecteurs voulaient en particulier "traiter sévèrement " était l'émigration elle-même. Tout au long de l'année 1855 et en 1856 en particulier, une série de lettres furent échangées entre un grand nombre de correspondants. Shadd en commenta beaucoup, décomposant les arguments ou les fautes de logique et les critiques restées sans réponses, et soulevant des points de controverse auxquels ses correspondants pouvaient répondre, tout comme elle l'avait fait avec les lettres WardFoote. L'un des arguments les plus tenaces des anti-émigrationnistes était que si « l'élévation» du peuple pouvait être atteinte par «la pratique de l'économie » et la "diffusion de la connaissance", "l'effort et l'éducation qui les rendraient capables d'émigrer [au Canada] leur permettrait également de rester [aux Etats-Unis]. « Si nous émigrons tels que nous sommes ", affirmait l'un des correspondants, "l'effort à faire pour élever notre condition ne serait pas diminué par un simple changement de localité. Les véritables remèdes à nos maux sont un juste partage des richesses du pays, de l'information et l'UNION» (2 mars 1855).

Consciente des limites de ces libertés particulières et confrontées une fois de plus à l'argument trompeur de "l'Union", Shadd décida d'en appeler à la conscience de ses lecteurs en confrontant les relations d'une «Union» économiquement et intellectuellement prospère avec les institutions, les politiques et les lois liées au gouvernement américain. Comme Shadd le rappelait une fois de plus à ses lecteurs, celui-ci offrait aux gens de couleur peu de liberté politique, voire même aucune :

Considérez calmement et sans préjugé votre position anti-émigration, mes frères, et vous aussi qui êtes indifférents! Non seulement vos mains sont liées contre votre propre salut par la servitude politique, mais vous encouragez un peuple à rester, et vous restez et accumulez de la richesse, les impôts mêmes qui sont non seulement 
versés aux Trésor des Etats-Unis et utilisés pour maintenir un gouvernement cimenté par le sang de vos frères, mais dont les législateurs s'approprient en fait une portion pour vous envoyer en Afrique; vous êtes donc forcés de partir, de payer en partie les dépenses de votre propre expatriation, et de verser votre quota [...] aux gardiens des limiers canins et humains, qui recherchent les fugitifs en fuite (5 juillet 1856). recherche des quelques libertés qu'il était en mesure de briguer, aidait à perpétuer une Union pro-esclavagiste plutôt qu'à l'entraver. Elle les fustigeait pour leur fausse conscience: «En vérité, mes frères, votre responsabilité est terrible! Mais elle est encore amplifiée par l'opposition que vous montrez contre ceux qui veulent renverser cet ordre des choses ». Et d'exiger : "Cessez de maintenir le gouvernement des EtatsUnis, s'il maintient l'esclavage humain et aussi longtemps qu'il le fait » (5 juillet 1856).

Le renoncement de Shadd est important ici : il ne signifie pas un abandon des valeurs républicaines américaines, mais plutôt une critique sévère d'un peuple et d'institutions qui n'incarnaient pas ces valeurs et peut-être n'étaient pas en mesure de le faire. Pour un abolitionniste, devenir citoyen canadien était un moyen de rester engagé dans l'idéal révolutionnaire d'autogestion à travers une conception plus large du Peuple, puisque pour les Canadiens aussi, «nous avons le devoir à l'égard de notre Constitution et de notre Gouvernement de nous rendre aptes à nous acquitter de [nos] devoirs d'hommes libres» (Provincial, 24 mars 1854). Cet entraînement à s'acquitter de ses devoirs participait d'un héritage global qu'un des pères fondateurs, Thomas Jefferson, avait appelé l'« épreuve de notre expérience ", celle-ci consistant à voir si l'on peut confier aux hommes de se gouverner eux-mêmes (Jefferson 1802 : 797). Il avait pour les Noirs libres du Canada une résonance particulière: "Nous n'avons jamais été des hommes libres auparavant, et avons donc besoin d'acquérir la capacité aussi bien de préserver nos droits et intérêts que de remplir nos devoirs » (Shadd, Provincial, 25 mars 1854). Très importantes sont ici les relations très étendues, voire transnationales dans lesquelles Shadd entendait placer les individus; elle affirmait que " comme l'influence [mutuelle] des nations n'est pas régionale mais mondiale", que "parmi celles des Amériques, la nôtre [le Canada] est en train d'acquérir une importance prédominante, de par sa position, ses relations avec les gouvernements européens [,] et son origine nationale, il est de première importance qu'un sentiment moral fort soit créé ici » (25 mars 1854). Ce n'est pas un hasard si la lettre ouverte est devenue pour Shadd le lieu de la création de ce «sentiment moral », ni qu'une si grande part de son action politique et les racines de sa pensée s'y soient développées. Les propriétés formelles de la lettre ouverte portent en elles jusqu'à ce jour ces questions : Quelles sont nos relations et nos engagements les uns envers les autres et, donc, envers nos institutions? La forme de la lettre permet de se confronter à ces questions à travers de vastes espaces géographiques, et même à travers de larges divisions idéologiques.

Ce qui est illustré avec force dans la rhétorique épistolaire de Shadd est l'effort de médiation qu'elle met en place par rapport à la conscience individuelle et collective de ses lecteurs - une médiation par laquelle on les invite à « se rendre compte de la façon dont des individus, pris dans la tourmente de leur expérience quotidienne, ont souvent une conscience erronée de leur position sociale » (1959:4). En prenant conscience de ces relations, "par leur réflexion et leur sensibilité ", les individus acquièrent une nouvelle façon de penser, expérimentent une transvaluation des valeurs » (Mills 1959: 8). Cette évaluation n'est pas ce que nous pourrions comprendre aujourd'hui comme 
une "critique des valeurs morales » nietzschéenne. Bien que Shadd ait appréhendé l'échange à travers lequel "la valeur de ces valeurs doit elle-même être remise en question", sa réévaluation ne cherchait pas à instituer une inversion des hiérarchies (Nietzsche, 5, souligné dans l'original). Pour elle, la transvaluation signifiait une reconfiguration de tout un ensemble de relations complexes, mettant en avant l'échange et refusant les relations de pouvoir purement verticales. Le but était la Liberté, créée au travers de ce qui pouvait être compris, selon les termes de la rhétoricienne Donna Haraway, comme un "réseau historique et hétérogène ", au travers duquel on pourrait commencer à "établir le narratif d'une politique possible d'articulation plutôt que de représentation » (1992:311).

La structure de l'échange discursif fondée sur les-moyens-et-la-fin mise en place par Shadd privilégiait emphatiquement une politique du Peuple et pour le Peuple. C'est dans cette structure que la lettre a pu devenir un véritable laboratoire de valeurs, sous l'obligation - et la promesse - de la liberté.

\section{BIBLIOGRAPHIE}

Bitzer, Lloyd. 1998 [1981]. « Political Rhetoric », Farrell, Thomas B. (ed). Landmark Essays on Contemporary Rhetoric (Mahwah NJ: Lawrence Erlbaum)

Browne, Stephen Howard. 2009. " Arts of Address in Revolutionary America », Lunsford, Andrea (ed). The Sage Handbook of Rhetorical Studies (Los Angeles: Sage)

Constitution of the United States. (1787) 2010. [En ligne : http://www.archives.gov/exhibits/ charters/constitution.html]

De Bow, J.D.B. 1854. Statistical View of the United States (Washington: Beverly Tucker, Senate Printer)

Hall, Stuart. 1996. « On Postmodernism and Articulation: An Interview with Stuart Hall», Grossberg, Lawrence (ed). Stuart Hall: Critical Dialogues in Cultural Studies (New York: Routledge)

Haraway, Donna. 1992. « The Promises of Monsters: A Regenerative Politics for Inappropriate/d Others », Grossberg, Lawrence, Nelson, Cary \& Paula Treichler (eds). Cultural Studies (New York: Routledge)

Henkin, David M. 2006. The Postal Age: The Emergence of Modern Communications in NineteenthCentury America (Chicago: The University of Chicago Press)

Jefferson, Thomas. 1900 [1802]. John P. Foley (ed). The Jefferson Cyclopedia (New York: Funk \& Wagnalls Company)

John, Richard R. 1995. Spreading the News: The American Postal System from Franklin to Morse (Cambridge: Harvard University Press)

Kielbowicz, Richard B. 1989. News in the Mail: The Press, Post Office, and Public Information, 1700-1860s (New York: Greenwood Press) 
King Jr, Martin Luther. 1986 [1963]. J.M. Washington (ed). I Have a Dream: Speeches and Writing that Changed the World (San Francisco: Harper)

Knowles, James D. (1834). Memoir of Roger Williams, The Founder of the State of Rhode Island (Boston: Lincoln, Edmands and Co)

LaClau, Ernesto \& Chantal Mouffe. 1985. W. Moore \& P. Cammack (trans). Hegemony and Socialist Strategy: Towards a Radical Democratic Politics. (London: Verso)

Logan, Shirley Wilson. 2008. Sites of Rhetorical Education ion Nineteenth-Century Black America (Carbondale: Southern Illinois University Press)

McGee, Michael. 1988 [1981]. « The Ideograph », Farrell, Thomas B. (ed). Landmark Essays on Contemporary Rhetoric (Mahwah NJ: Lawrence Erlbaum)

Mills, C. Wright. 2000 [1959]. The Sociological Imagination. Fortieth Anniversary Edition. (London: Oxford University Press)

Nietzsche, Friedrich Wilhelm. 1998 [1887]. Maudemarie, Clark \& Alan J. Swensen (eds). On the Genealogy of Morality: A Polemic (Indianapolis: Hackett)

Nussbaum, Martha. 2008. Liberty of Conscience: In Defense of America's Tradition of Religious Equality (New York: Basic Books)

Perelman, Chaim \& Lucie Olbrechts-Tyteca. 1958. Traité de l'argumentation. La nouvelle rhétorique (Bruxelles : Éditions de l'Université de Bruxelles)

Rhodes, Jane. 1998. Mary Shadd Cary: The Black Press and Protest in the Nineteenth Century (Bloomington: Indiana University Press)

Shadd, Mary Ann. 2 November 1851. Letter to George Whipple. American Missionary Association Papers. (New Orleans: Amistad Research Center)

Stormer, Nathan. 2004. "Articulation: A Working Paper on Rhetoric and Taxis », Quarterly Journal of Speech 90:3, 257-284

Tocqueville, Alexis de. 2000 [1835]. DemocracyinAmerica (Chicago: University of Chicago Press)

Williams, Roger. 1867 [1644]. Caldwell, Samuel L. (ed). The Bloudy Tenet of Persecution (Providence: Providence Press Co., Printers)

\section{NOTES}

1. Le Bureau du recensement évalue le nombre des gens de couleur libres en 1840 et en 1850 à respectivement 386.303 et 434.495 ; la population des esclaves en 1840 et en 1850 à respectivement 2.487.455 et 3.204.313. Voir De Bow $1854: 63,82$.

2. Ici l'acception de sens est plus large que dans « lettre ouverte».

3. Pour plus de détails sur l'histoire de la poste et ses relations avec les journaux et les mouvements réformistes, voir John, Kielbowicz \& Henkin.

4. Je dois beaucoup à Jennifer A. Lutman pour nos échanges sur la théorie de l'articulation dans les études culturelles. Voir les travaux de Hall, LaClau \& Mouffe, Haraway\& Stormer.

5. Les détails biographiques sur la vie de Mary Shadd sont essentiellement tirés du livre de Rhodes.

6. Les droits de citoyenneté canadienne étaient limités pour les femmes et les personnes sans propriétés qui ne possédaient pas encore le droit de vote. 
7. Les détails biographiques concernant Williams proviennent de deux sources principales: Knowles et Nussbaum.

\section{RÉSUMÉS}

Cet article examine les lettres ouvertes de la réformiste anti-esclavagiste Mary Ann Shadd, au prisme des valeurs considérées à la fois comme moyens et comme fins. Il met l'accent sur la nature énonciative de la rhétorique épistolaire de l'auteur, et sur le pouvoir rhétorique de la lettre ouverte considérée comme genre relevant de l'examen de conscience à l'intérieur des structures d'échange publiques. Portant une attention critique à la conception de la critique et de la dissension chez Mary Ann Shadd, cette étude présente la lettre publique comme un moyen utilisé par les abolitionnistes pour réaliser leur travail de médiation pour la collectivité et énoncer une transvaluation des valeurs.

By applying the lens of values as means and ends, this essay examines the public letters of antislavery reformer Mary Ann Shadd. It focuses on the articulative nature of Shadd's epistolary rhetoric, and the rhetorical power of the public letter to serve as a genre of conscience within public structures of exchange. In drawing critical attention to shadd's embrace of critique and dissent, the essay figures the public letter as a means and end for accomplishing the anti-slavery work of mediating a collectivity and articulating a transvaluation of values.

\section{INDEX}

Keywords : critique, dissent, epistolary rhetoric, public letter, value

Mots-clés : critique, dissension, lettre ouverte, rhétorique épistolaire, valeur

\section{AUTEURS}

\section{SUZANNE B. SPRING}

Colgate University 